\title{
CD4 memory $T$ cell levels predict life span in genetically heterogeneous mice
}

\author{
RICHARD A. MILER,,$;, 1$ CLARENCE CHRISP, ${ }^{\dagger}$ AND ANDRZEJ GALECKI ${ }^{\ddagger}$ \\ *Department of Pathology, ' Unit for Laboratory Animal Medicine, ${ }^{\ddagger}$ Institute of Gerontology, and \\ *Ann Arbor DVA Medical Center, University of Michigan, Ann Arbor, Michigan 48109-0642, USA
}

\begin{abstract}
Aging leads to changes in the relative proportions of several functionally distinct $T$ cell subsets, including increases in the proportions of memory cells in the CD4 and CD8 subsets and in the proportion of $T$ cells expressing the multiple-drug resistance pump P-glycoprotein. To see whether individual differences in $\mathbf{T}$ cell subset levels predict life span, we measured the levels of five age-sensitive $T$ cell subsets, at 8 and again at 18 months of age, in the peripheral blood of genetically heterogeneous mice bred as the progeny of CB6F1 females and C3D2F1 males. The strongest immunological predictor of life span in univariate regression analyses was the proportion of CD4 memory cells measured at 18 months of age $(P=0.003)$. CD4 memory cell levels remained strongly correlated with life span $(P<0.0003)$ in a multiple regression analysis after adjustment for sex. The proportion of CD4 cells expressing P-glycoprotein was also correlated with life span $(P<0.01)$, but only in male mice. Weaker relationships were observed between life span and 8month tests of CD8 memory and CD8 P-glycoprotein levels, for CD4 naive cells at 18 months, and for the change in CD4 naive cells between 8 and 18 months of age; these were, however, near the margin of statistical significance and could reflect chance relationships. The relationship between CD4 memory cell levels and life span was similarly strong regardless of the cause of death in mice whose death was attributable to lymphoma, fibrosarcoma, mammary carcinoma, and other forms of terminal pathology. Additional work is needed to discriminate between two hypotheses: 1) that high levels of CD4 memory cell themselves predispose to disease and early death, particularly from neoplasia; or 2) that accumulation of CD4 memory cells is a biomarker of some underlying process-perhaps accelerated aging-that itself leads to early mortality.-Miller, R. A., Chrisp, C., Galecki, A. CD4 memory T cell levels predict life span in genetically heterogeneous mice. FASEB J. 11, 775-783 (1997)
\end{abstract}

Key Words: $T$ lymphocytes $\cdot$ memory $T$ cells $\cdot$ aging $\cdot$ mice $\cdot$ longevity

THE RELATIONSHIPS AMONG IMMUNE STATUS, aging, and cancer are complex and controversial. Although it is clear that for most kinds of cancer the incidence of risk increases dramatically in the second half of the life span, the suggestion that diminished immune responsiveness contributes to the increased cancer risk of the elderly is highly controversial. Patients whose immune systems are weakened by congenital abnormalities, ablative chemotherapy or radiation therapy, or HIV infection are indeed put at higher risk for cancer than normal controls, but the forms of neoplasia typically seen in these settings are unusual, restricted typically to hematopoietic malignancies and a few other tumors not commonly seen in the elderly. The speculation that the immunodeficiency of aging might contribute to increased tumor incidence in aged individuals rests at present on indirect and unconvincing evidence. A few studies (14) of very old humans have suggested that immune anergy may be associated with an increased risk of mortality in fairly short periods of follow-up (rarely longer than $5 \%$ of the mean life span); however, these studies have not discriminated between neoplasia and other causes of death, and have often either failed to reach statistical significance (3) or to adjust for the confounding effects of age on both anergy and life expectancy $(1,2)$. Because people are so long-lived, it is very expensive to perform studies in which a substantial fraction of the life span elapses between a potentially prognostic test and the diagnosis of a lethal illness; studies with only shortterm follow-up are potentially confounded by the possibility that the illness itself may have altered the outcome of the test in question.

In mice and humans, aging leads to progressive changes in $\mathrm{T}$ lymphocyte subsets. In both species, memory $\mathrm{T}$ cells increase in their relative proportions whereas naive or virgin $T$ cells decline in parallel (5$8)$. The shift from virgin to memory $T$ cell subsets affects both the CD4 and CD8 T cell subsets. In mice, there is also an increase with age in the proportion of CD4 and CD8 T cells that express the plasma membrane pump P-glycoprotein; the increase in these CD4P and CD8P cells affects both the naive and

\footnotetext{
${ }^{1}$ Correspondence: Geriatrics Center, MSRB-3 Room 6301, Box 0642, 1150 W. Medical Center Dr., Ann Arbor, MI 481090642, USA.
} 
memory cell subsets (9). The accumulation of memory cells and of cells that express P-glycoprotein may account for some elements of age-associated decline in $\mathrm{T}$ cell proliferation and cytokine production in vitro $(6,10-12)$, but it is not yet clear whether or in what way $\mathrm{T}$ cell subset shifts contribute to age-associated changes in in vivo immune responses. Nevertheless, the changes with age in $T$ cell subset distribution, together with the demonstrable differences between subsets in in vitro responsiveness, suggest the testable hypothesis that measurements of $\mathrm{T}$ cell subset distributions might provide information about interindividual differences in protective immunity and susceptibility to late-life illnesses, including neoplastic diseases.

To test this idea, we carried out a study of $\mathrm{T}$ cell subsets, longevity, and pathology in mice. Although most previous work on aging mice has involved genetically homogeneous groups of animals-typically, inbred lines or their F1 hybrids-we chose instead to use mice bred via a four-way cross-breeding scheme (13) that can produce an arbitrarily large number of genetically distinct mice, all of which share the same four grandparents and are thus the genetic equivalent of one extremely large sibship. We report here that one of the $T$ cell subset tests-specifically, the proportion of $\mathrm{CD} 4$ cells expressing the memory cell marker CD44 in 18-month-old mice-has a significant ability to predict longevity. Consistent with our hypothesis, mice with high levels of CD4 memory cells-whose immune system thus resembles that of older mice-tend to have relatively short life expectancy compared to sibs with lower levels of CD4 memory $\mathrm{T}$ cells. The relationship between CD4 memory cell levels and longevity holds for mice whose death is attributable to any of several kinds of neoplasia including lymphoma, fibrosarcoma, and other cancers.

\section{MATERIALS AND METHODS}

\section{Mice and husbandry}

Mice for this study were the progeny of a cross between CB6F1 females and C3D2F1 males; each mouse in the group is thus genetically distinct, but shares all four grandparents with every other mouse. The population is thus the genetic equivalent of a very large sibship. We refer to the offspring of this genetic cross as UM-HET3 mice. They were housed in same-sex groups in one room of a specific pathogen-free colony. Each cage was protected by a microisolator top to minimize cage-to-cage spread of infectious agents. To verify the pathogen-free status of the colony, sentinel mice (not part of the experimental group) were exposed to spent bedding collected from all of the test mice, and then later tested serologically for antiviral titers and evidence of pinworm. These screening tests were carried out every 3 months and were repeatedly negative throughout the course of this study. Mice were given free access to rodent chow and water. Mice were inspected at least once/day (twice/day except on weekends) and weighed monthly. Mice judged to be potentially ill were placed on a "watch list" and were weighed at least weekly and inspected at least twice a day 7 days/wk.

\section{Exclusion criteria}

The cohort of mice used for this analysis originally contained 173 animals, of which 90 were male and 83 were female. These mice were all born between September 14, 1993 and February 16, 1994. Six cages containing male mice were eliminated from the study because fighting had led to bite wounds; all 22 males in such cages were culled at ages of less than 12 months, and these animals are not included in the statistical analyses. In addition, 18 males were found, at necropsy, to be suffering from mouse urinary syndrome ("MUS"), , a syndrome characterized by obstruction of the urethra with expansion and (in extreme cases) rupture of the urinary bladder, which has previously been observed in group-housed males of several inbred strains $(14,15)$ and is thought to represent a response to the social stresses that arise in all-male groups. These males were also excluded from all analyses; their mean age at death was $15.6 \pm 4.2(\mathrm{SD})$ months. Four other males were also excluded from the study: one because it was killed in error at the age of 18.7 months, and three others because they died spontaneously before 8 months of age and thus did not yield any immunological data. The population of mice in the study included 46 males and 83 females, among which 41 males and 79 females survived to the 18-month assay point.

\section{Assessment of $\mathbf{T}$ cell subsets}

These procedures have been described in greater detail elsewhere (16). In brief, $400 \mu$ l blood samples were taken from each mouse at 8 months of age and again at 18 months. Each sample was divided into eight aliquots and tested for $\mathrm{T}$ cell subset representation by using combinations of monoclonal antibodies and/or fluorescein-tagged latex beads. Table 1 summarizes the eight subsets tested at each age. A small percentage of the assays were deemed to be unreliable on technical grounds and these were not used in the analyses.

\section{Necropsy}

Mice were judged to be moribund if, in the judgment of an experienced technician, they were considered unlikely to survive for more than another few days. This assessment was based on a constellation of signs including continued or severe weight loss, hunched posture, poor grooming, failure to eat or drink, extreme lethargy or partial paralysis, or the presence of a large ulcerated tumor. Mice judged to be moribund were killed and subject to necropsy, as were mice found dead in their cages. All of the mice killed when moribund were found at necropsy to have pathological signs of advanced illness. The adoption of this "sacrifice when moribund" strategy greatly increases the proportion of animals that yield highquality necropsy data, but poses the potential risk of underestimating the life span in animals killed before their natural death. The mean age at death of mice killed when moribund was 28 months ( $S D=4$ months; $N=85$ ), which is significantly higher than the age at death of those found to die spontaneously ( $25 \pm 6 ; \mathrm{N}=44 ; \mathrm{F}=9.00 ; P=0.003$ by the Student-NewmanKeuls test). The difference in life span between these two groups of mice might reflect differences in age of incidence between diseases that kill quickly and those that lead to gradual morbidity before demise. The data do not, however, sup-

\footnotetext{
${ }^{2}$ Abbreviations: MUS, mouse urinary syndrome; CD4M[8], refers to the CD4M determination made at 8 months of age.
} 


\begin{tabular}{lll}
\hline \hline Antibody & \multicolumn{1}{c}{ Description } & \multicolumn{1}{c}{ Calculation } \\
\hline CD3 & T cells & Cell number per microliter of blood \\
CD4 & Class II restricted (“Helper”) T cells & CD3(+), CD4(+) as percentage of CD3 \\
CD8 & Class I restricted (“cytotoxic") T cells & CD3 $(+)$, CD8(+) as percentage of CD3 \\
CD4M & Memory CD4 cells & CD4 $(+)$, CD44(+) as percentage of CD4 \\
CD8M & Memory CD8 cells & CD8 $(+)$, CD44(+) as percentage of CD8 \\
CD4V & Virgin CD4 cells & CD4 $(+)$, CD45RB(low) as percentage of CD4 \\
CD4P & P-glycoprotein(+) CD4 cells & CD4 $(+)$, R123-extruding as percentage of CD4 \\
CD8P & P-glycoprotein(+) CD8 cells & CD8 $(+)$, R123-extruding as percentage of CD8 \\
\hline
\end{tabular}

port the idea that this strategy leads to a serious underestimation of longevity.

Details of the methods used to assess pathological lesions have been described in detail earlier (13). All slides were examined and all were diagnoses made by the same pathologist (C. Chrisp). Although most mice had various conditions not usually seen in young animals, it was almost always possible for the pathologist to identify one (or occasionally two) form of serious illness that very likely caused death or, for moribund animals, the severe illness that had led to the death of the mouse. The necropsy data were used to divide the mice into subgroups according to major cause of death. These subgroups consisted of death due to 1) fibrosarcoma; 2) lymphoma; 3) mammary tumor; 4) other forms of neoplasia; 5) nonneoplastic illness; and 6) cause undetermined. The lymphoma group included several histologically distinct forms such as follicular center cell, small lymphocytic, and lymphoblastic lymphomas. The "other neoplastic" group included cases of pulmonary adenocarcinoma, hepatocellular carcinoma, histiocytic sarcoma, pituitary adenoma, and several rarer lesions. Nonneoplastic illnesses included congestive heart failure, glomerular amyloidosis, endometritis, jejunal intussusception, middle ear hemorrhage, and pyometra. Of the 11 mice in the "undetermined" group, five had suffered severe postmortem autolysis; in the other six, it proved impossible to assign a probable cause of death from among the multiple lesions found at necropsy.

\section{Statistical analysis}

The dependent variable used in the analysis was life span. Independent variables included gender and $T$ cell subsets measured at 8 and 18 months. When the analysis was performed, one mouse was still alive (censored observation) and it was excluded from calculations. The analysis was performed in two steps. In the first exploratory step, simple univariate regression models were considered to study the effect of gender and $T$ cell subsets on life span. In the second step, several multiple regression models were considered that correspond to a classical analysis of covariance. The second stage allowed us to take into account the potential confounding effect of gender on the relationship between $\mathrm{T}$ cell subsets and life span. In addition, interaction terms between gender and various CD4 memory $\mathrm{T}$ cell variables allowed us to determine whether the relationship between the immunologic variables and life span was the same in both gender groups.

A similar approach was used to study the relationship between CD4M[18] and life span in subgroups differing in their cause of death. To develop the best model (life span $=$ sex $+\operatorname{cd} 4 \mathrm{M}[18])$, several pairs of nested models were compared using F-test statistics. The model fit was checked for individual and systematic departures of the observed and fitted values by using informal (e.g., inspection of residuals) and formal methods [e.g., based on score tests for extra parameters (17)].
Calculations were performed by using PROC REG and PROC GLM in SAS/STAT. Values of $P<0.05$ were considered to be significant.

\section{RESULTS}

Our working hypothesis was that one or more of the $T$ cell subset markers would prove to have prognostic significance, and specifically that there would be a relationship between the marker (or markers) and life span. Some of the $T$ cell subsets-the CD4M, CD8M, CD4V, CD4P, and CD8P populations-have been shown in previous cross-sectional analyses to change with age. Since CD4M, CD8M, CD4P, and CD8P all increase with age, our prediction was that mice with high values of these cells at any given age would have a relatively short life span. CD4V values tend to decline with age, and we therefore hypothesized that low CD4V values would be correlated with a short life span. Although reports of the effects of age on mouse CD4 levels are less consistent, several published studies $(18,19)$, including our own of a separate UM-HET3 population (20), have shown a decline with age in the proportion of CD4 cells in mouse blood; we therefore predicted that if CD4 levels showed a relationship with life span, it would be one in which low CD4 levels predicted short life span.

To explore these ideas, we first calculated the regression coefficients for each of the 16 subset measurements (eight tests at each of two ages). Table 2 shows the calculated regression coefficients, $t$ statistics, and the significance of the effect in a two-tailed procedure for all cases in which $p(t)<0.10$. The strongest predictor among the immune status tests was CD4M[18]. The regression coefficient, -2.43 , had a sign consistent with the hypothesis that large CD4M[18] values would be correlated with short life span. Sex was also a significant predictor, with female mice longer-lived than males. There were also marginally significant relationships between life span and CD8M[8], CD8P [8], CD4P [18], and CD4V[18], and with the change in CD4V between 8 and 18 months of age; these had much less predictive power, and some are likely to represent chance relationships often seen in a series of multiple comparisons. The pa- 
TABLE 2. Predictors of lifespan by univariate regression

\begin{tabular}{|c|c|c|c|c|}
\hline Variable & Time & $\begin{array}{l}\text { Regression } \\
\text { coefficient }\end{array}$ & $\mathbf{t}$ & $p(t)$ \\
\hline $\operatorname{Sex}^{a}$ & $(\mathrm{NA})^{b}$ & -88 & -3.35 & 0.0011 \\
\hline CD8M & 8 & -2.28 & -1.91 & 0.059 \\
\hline CD8P & 8 & 2.04 & 1.95 & 0.054 \\
\hline CD4M & 18 & -2.43 & -3.05 & 0.0029 \\
\hline CD4P & 18 & -1.79 & -1.93 & 0.056 \\
\hline CD4V & 18 & 1.22 & 1.71 & 0.090 \\
\hline$\Delta \mathrm{CD}^{\prime} \mathrm{V}^{\mathrm{c}}$ & (NA) & 2.25 & 1.96 & 0.056 \\
\hline
\end{tabular}

rameter estimates for CD4M, CD8M, CD4P, and $\mathrm{CD} 4 \mathrm{~V}$ have the sign predicted by the hypothesis, since the former three subsets increase whereas CD4V cells decline with age. Our working hypothesis predicted that high CD8P levels should be correlated with short life span, but we note that the marginally significant relationship between CD8P and life span (Table 2) is opposite to the predicted direction. These univariate calculations are exploratory; the results are not adjusted for multiple comparisons, and so require cautious interpretation.

To see whether stratification by sex might reveal relationships between $\mathrm{T}$ cell markers and life span that had been obscured by combining males with females for the initial regression estimates, we plotted life span vs. $T$ cell subset levels separately for males and females for each of the $16 \mathrm{~T}$ cell assays. In most cases, this recalculation of the regression results did not reveal significant relationships not already apparent from analysis of the pooled data. The exception was the relationship between CD4P [18] and life span, which was much stronger in male than in the female mice: for males the regression coefficient for $\mathrm{CD} 4 \mathrm{P}[18]$ was $-4.38(\mathrm{t}=-2.72 ; P=0.01)$, whereas for females the regression coefficient was $0.20 \quad(t=0.18$; $P=0.86$ ).

Figure 1 shows the scatterplots for the six relationships that were at least marginally significant (at $P<0.1)$. The scatterplots show that the relationships suggested by the coefficients collected in Table 2 appear to apply to both males and females (except for the CD4P [18] values, as noted above) and that the relationships are not attributable to a small number of unusual outlying individuals.

We next used multiple linear regression to calculate the best fit of our data to a model in which sex and $T$ cell determinations predict longevity. The bestfit model yielded $F=11.96(P<0.0001)$. Only sex and CD4M[18] met the threshold for inclusion in the model. Table 3 shows the best-fit parameters and their statistical significance. $\mathbf{R}^{2}$ for the model was 0.18 ; thus, $18 \%$ of the variance in life span could be accounted for by differences among mice in sex and $\mathrm{CD} 4 \mathrm{M}[18]$.
The calculations just presented suggest that high CD4M levels at 18 months of age are correlated with relatively low life expectancy. It is possible that such a relationship might reflect an effect of CD4M levels on the development of a specific kind of late-life disease. Alternately, high CD4M levels might be caused by the early stages of some specific disease process. To test these ideas, we calculated the regression coefficients for CD4M[18] by using the multiple regression procedure illustrated in Table 3 separately for mice in each of five groups stratified by principal cause of death. The results are shown in Table 4, which presents the regression coefficients after adjustment for sex. The parameter estimates for each of the three distinct neoplastic causes of death and for the "other neoplasia" category are similar to one another and to the coefficient $(-2.88)$ calculated for the entire group of mice. This suggests that $\mathrm{CD} 4 \mathrm{M}[18]$ has an approximately equal effect on life span regardless of the cause of death, at least for deaths attributable to neoplasia. The parameter estimate for the small number of mice whose deaths were attributable to nonneoplastic lesions $(-1.65$; $\mathrm{N}=13$ ) is consistent in sign with the other values shown in Table 4, but lower than that for the other categories; it does not approach statistical signifcance. A multiple regression model in which life span was estimated on the basis of sex, CD4M[18], a term indicating the presence or absence of neoplastic death, and the [CD4M $\times$ neoplasm] interaction term yielded an estimate for the $\operatorname{CD} 4 \mathrm{M}$ [18] parameter $(-2.37 ; t=-2.46 ; p(t)=0.016)$ not greatly different from that shown in Table 3 . The interaction term itself was not significant (parameter $=-0.54$, $\mathrm{t}=-0.28 ; \mathrm{p}(\mathrm{t})=0.78)$, consistent with the idea that the relationship of life span with CD4M[18] was not significantly different in mice that did or did not develop a fatal neoplasm; however, the sample size is small enough that an effect of this kind might have gone undetected. The term for neoplastic death did not have any significant effect in this model, i.e., it did not contribute significantly to the variance in life span. Additional studies, including larger numbers of animals with other causes of death, will be needed to decide whether CD4M[18] values have prognostic significance for nonneoplastic deaths.

\section{DISCUSSION}

Several previous attempts have been made to predict longevity or disease risk by tests of age-sensitive immune status indices. The long life of humans makes it difficult to perform a study in which more than a small fraction of the life span elapses between the time of testing and the eventual demise of the tested subjects. Several studies of very old humans have documented a relationship between skin test anergy (1) 

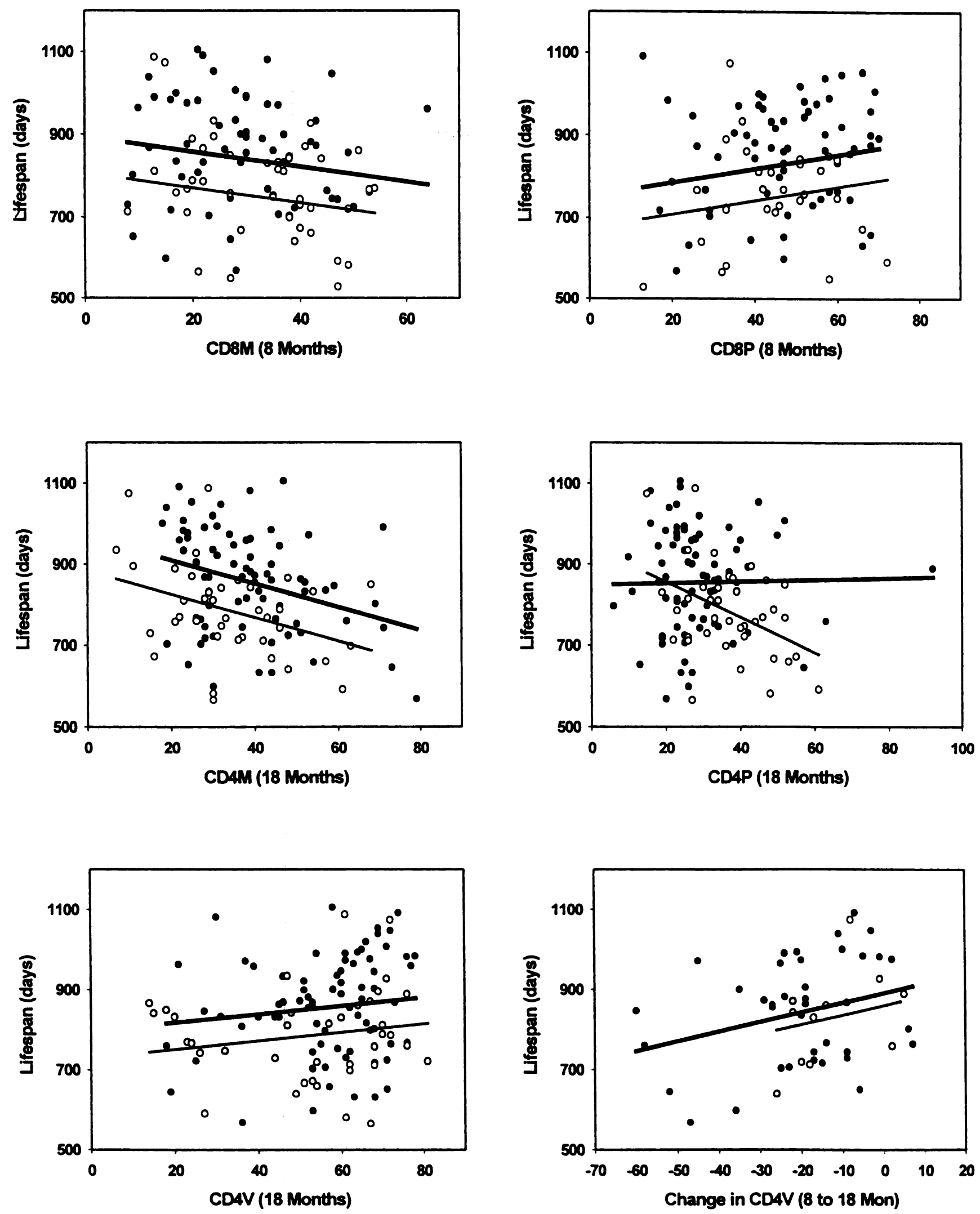

Figure 1. Plots of $\mathrm{T}$ cell subset counts against lifespan for the assays listed in Table 2. Each point represents a single mouse. Males are shown as open circles and females as filled circles. The lines shown (bold for females) are derived from the multiple regression equation calculating life span as a function of sex, the $T$ cell subset, and, where significant, the [sex $\times$ subset] interaction term. 
TABLE 3. Predictors of lifespan using multiple regression

\begin{tabular}{lccc}
\hline \hline Variable & Regression coefficient & $\mathrm{t}$ & $\mathrm{p}(\mathrm{t})<$ \\
\hline Sex $^{a}$ & -84.1 & -3.69 & 0.0004 \\
CD4M[18] & -2.88 & -3.77 & 0.0003 \\
\hline
\end{tabular}

${ }^{a}$ Male $=1 ;$ female $=0$.

or poor mitogen responsiveness (2) and low subsequent survival.

The first of these studies was based on a cohort of 52 nursing home residents, all of whom were more than 80 years of age, and included only a 2-year follow-up period; none of the 34 deaths were attributable to cancer. The second study monitored 403 elderly apartment-dwellers, initially of average age 86 , for a 25-month follow-up; seven of the 61 deaths were due to malignancy. Neither study tested the hypothesis that the relationship between immune nonresponsiveness and mortality risk reflects the common dependence of both results on the age of the subject, and neither study convincingly refutes the idea that preexisting illness may have contributed both to poor immunity and high mortality risk. A third group (4) has reported an association between poor 2-year survival and a cluster of immune indices (high numbers of $\mathrm{CD} 8$ cells, low numbers of CD4 cells, low B cell numbers, and low responses to $\mathrm{T}$ cell mitogens) in a group of 102 very old individuals (age range 86-92). A fourth study (3) tested the relationship between skin tests for delayed hypersensitivity and longevity in a group of 293 apparently healthy elderly persons (average age of 72 years), with a 9-year follow-up period. Skin test anergy predicted relatively poor survival, but the effect was of marginal significance $(P=0.06)$ after adjustment for subject age (95\% confidence interval 0.94-3.79 for all-cause mortality). In this population, there was also a trend for anergy to be associated with increased risk of death from cancer (relative risk 2.65; $95 \%$ confidence interval 0.74-9.55); there was little evidence for an association between anergy and increased risk of cancer incidence (relative risk of 1.15). Together, these reports suggest that poor immune responsiveness may be associated with low survival in very old humans, but more information is needed about the robustness of the effect, the influence of potential confounders (sex, age, preexisting illness), effects on specific forms of illness (including neoplasia), and the prognostic value of tests administered to younger individuals.

In principle, similar questions could be explored in rodents well before the end of their life span, but the difficulties in assessing immune status without harm to the animal have been an obstacle. Boersma et al. (18) have reported that the proportion of CD8(+) cells among peripheral blood $T$ cells predicted remaining life span in CBA mice at any age tested; high levels of CD8 cells were associated with high mortality risk. No such relationship was observable in C57BL/ 6 mice, however, and the dramatic increase with age in the proportion of $T$ cells expressing the CD8 marker seen by this group (18) is not typical of most other studies of inbred (21) or genetically heterogeneous (20) mice. An interesting study (22) of mice bred as a backcross between genetically heterogeneous lines differing in early life humoral immunity reported that those animals with highest anti-erythrocyte antibody responses at $8 \mathrm{wk}$ of age were also likely to have the greatest longevity. Neoplasia was found in $18 \%$ of the 102 mice taken to necropsy; however, infectious disease is likely to have been a prominent cause of death in this conventional (i.e., not specific pathogen-free) colony, and thus the data do not provide much insight into possible links between immune responsiveness and late-life neoplastic disease.

A study of four-way cross mice (23) found that death from lymphoma within the first 18 months of life was associated with high levels of CD4M, CD8M, CD8, and CD4V cells tested at 6 months of age. This study, however, had several weaknesses. First, specific pathogen-free status was lost when the mice were 14 months old, and it is possible that the relationship between immunity and longevity may have been complicated by infection. Second, the grandparents used to generate the four-way cross included two strains, AKR/J and SJL/J, which themselves have an unusually high rate of neoplasia in early life. Third, the housing method used (one mixed-sex pair per cage) tends to shorten life span in the multiparous females; the relationship between immune markers and early tumor death could have reflected differences between males and females in both outcomes. Last, the results, though statistically significant, were based on only five deaths in a population of only $\mathbf{4 0}$ mice. The current protocol was designed in part to avoid these potential confounders by removing $A K R / J$ and $S J L / J$ from the breeding system, increasing the numbers of subjects of both sexes, using virgin animals, careful maintenance and documentation of specific pathogen-free status, and continuing the study until the death of all animals in the cohort.

The CD4M subset has been shown to increase with age in cross-sectional studies of mice $(5,6,19)$ and

TABLE 4. Prediction of lifespan by $C D 4 M(18)$ after adjustment for sex in subgroups with differing causes of death

\begin{tabular}{lcccc}
\hline \hline Cause of death & $\begin{array}{c}\text { Regression } \\
\text { coefficient }\end{array}$ & $\mathrm{N}$ & $\mathrm{t}$ & $\mathrm{p}(\mathrm{t})$ \\
\hline Fibrosarcoma & -3.92 & 17 & -1.92 & $\mathbf{0 . 0 7 6}$ \\
Lymphoma & -2.89 & 28 & -2.11 & 0.045 \\
Mammary carcinoma & -4.66 & 11 & -1.05 & 0.325 \\
All other neoplasms & -2.86 & 41 & -2.12 & 0.041 \\
Nonneoplastic causes & -1.65 & 13 & -0.59 & 0.567 \\
\hline
\end{tabular}


humans $(7,24)$. A longitudinal study of UM-HET3 mice also showed (20) increases in the CD4M subset in both male and female animals; the proportion of CD4 cells in the CD4M subset declined by 4.5 percentage points ( $\pm 2.2 \mathrm{SEM} ; \mathrm{N}=59$ ) between 8 and 14 months of age, and another $6.3( \pm 1.9 ; \mathrm{N}=48)$ percentage points between 14 and 20 months of age. Since high levels of CD4M cells are characteristic of older mice, we predicted that within a group of mice at a given age, those with the highest levels of CD4M cells would be more susceptible to late-life illness. The data show that high CD4M levels at 18 months of age are indeed characteristic of mice destined to die relatively soon. An increase of 14 percentage points in the CD4M level (one standard deviation) is associated in this population with a decline of $\mathbf{4 0 . 9}$ days of life expectancy, i.e., about $5 \%$ of the mean or median life span.

We can consider three classes of explanations for this correlation: 1) disease leads to changes in CD4M levels as well as life span; 2) CD4M levels reflect immune competence, which in turn alters incidence or progression of disease; and 3) CD4M levels reflect the pace of a process, such as aging, that influences disease risk and longevity.

The first of these ideas would attribute the accumulation of CD4M cells in some particular mice to an underlying disease state, such as neoplasia, that also leads to the relatively rapid death of the animal. Although this is hard to rule out entirely, we consider it unlikely for two reasons. First, the relationship between CD4M and longevity is seen in mice dying of diverse causes. Lymphomas, fibrosarcomas, and mammary adenocarcinomas are sufficiently common in these animals to justify stratification into separate classes; in each case, as well as for the large group of "miscellaneous" other neoplasms, the regression coefficient is of roughly equal size. The strength of the relationship may well be slightly less for deaths from nonneoplastic causes, but it will be difficult to test this idea until larger numbers of animals are evaluated. None of these illnesses is known to induce an increase in peripheral blood CD4M levels in mice or humans. It is possible that one of these forms of disease systematically increases $\mathrm{CD} 4 \mathrm{M}$ values through an unknown mechanism, but it seems less likely that all would do so and to a similar extent. Second, crosssectional studies of 18-month-old mice rarely find morphologic evidence of malignancy at this comparatively early age (25). The mean interval between blood sampling and death in this study was 9.6 months, or about one-third of the mean life span. Any preneoplastic process putatively responsible for the alteration in CD4M[18] levels would have to be sufficiently far advanced at $\mathbf{1 8}$ months to lead to the immune alteration, and yet sufficiently immature so as not to lead to death or severe illness for many additional months.
A second possible explanation for our findings would be that alterations in immune function, detectable by $\mathrm{CD} 4 \mathrm{M}$ counts, lead to an increased risk for tumor incidence or progression. The idea that poor immune function contributes to cancer risk (the immune surveillance hypothesis) has become less popular in the face of evidence that the spectrum of tumors seen in congenital or iatrogenic forms of immune deficiency is quite different from that seen in old age (26). There is, however, very little evidence for or against the idea that the form of immune deficiency seen in normal aging, including a shift in the proportions of memory and naive cells, would alter host defenses against neoplasia. Our correlational data, though consistent with a cause-and-effect relation between immunosenescence and neoplastic disease, certainly do not prove such a link; experimental protocols that modify memory cell number or function in animals that are then followed for tumor development will be needed to explore this issue further. There is some evidence that maintenance of immune function in aging mice by sequential repopulation by bone marrow and thymus transplantation can diminish mortality risk in mice, but the effect was seen only in the first half of the life span (27). The correlation between humoral immunity and life span in Biozzi backcross mice (22) is also consistent with this reasoning. Tests to see whether high CD4M levels predict poor responsiveness to immunogens in our genetically heterogeneous mice would provide insight into the relationship between this $\mathrm{T}$ cell subset and levels of in vivo immunity.

A third possible explanation would connect CD4M changes to life span by proposing that both are timed in part by some underlying factor such as a hypothetical rate of biological aging (28). Some gerontologists have argued that the aging process is too complex and multifactorial to be reduced to a single dimension (29-31), whereas others $(16,32,33)$ have argued that individual members of a species might age at measurably different rates, which can be defined and validated in an operationally useful way. One way to test these ideas would be to see whether measurements of age-sensitive tests in one physiological systemsuch as the immune system-might predict the outcome of tests in other systems such as, for example, muscle function, accumulation of molecular damage, or disease incidence. In one such study using 9- to 12-month-old UM-HET3 mice, high levels of CD4M were found to be correlated with low muscle strength and endurance (16); further work is needed to determine whether these relationships also apply to older animals and whether the muscle function tests themselves predict life span. A second study (33), using aging macaque monkeys, 
found that a composite variable-summarizing information on interindividual differences in several age-sensitive measures in different physiological systems - was influenced by blood antioxidant levels, which in turn influenced disease susceptibility. Data from these studies, although consistent with the idea that it may be possible to define aging rate in a useful way, are still fragmentary and inconclusive. Further tests of correlations between age-sensitive immune markers (such as CD4M) and the extent of change in other age-sensitive domains will help to support or refute this class of hypothesis.

Our univariate regressions suggested that other $T$ cell subsets may also provide prognostic information about life span, but the relationships were less strong that that seen for CD4M, and none of the other immune variables contributed significantly to the multiple regression equation. Analysis of a larger group of mice might provide sufficient statistical power to test these possible relationships more effectively. The ability of the CD4P test to predict life span in male mice, if it is replicated in an independent sample, might potentially give information about immune status beyond that available from CD4M measurements. Levels and rates of change of CD4P cells are correlated in UM-HET3 mice with CD8P cells, but not with CD4M or CD4V cells (20). P-glycoprotein expression subdivides the CD4 memory subset (9); CD4 memory cells that do express P-glycoprotein fail to respond in tissue culture tests of proliferation and cytokine production $(10,11)$. It is therefore plausible that high proportions of CD4P cells might lead to poor immune responsiveness, an idea that can be tested directly by immunization of mice that differ in CD4P counts.

The UM-HET3 population was chosen for this study rather than a more traditional inbred or F1 hybrid mouse strain for two principal reasons. First, we hoped that conclusions would be more robust than those obtained from a study of genetically identical animals, and thus more likely to apply to other kinds of mice and perhaps other species as well. Second, we hoped that genetic differences among the mice might influence both life span and $T$ cell indices in a coordinated way. Our preliminary analyses (A. U. Jackson, R. A. Miller, and D. T. Burke, unpublished data) indicate the presence of genetic variation in UM-HET3 mice with significant influence on CD4M [18] and CD8P [18] levels, and it will be interesting to see whether variation at these loci also predicts longevity.

We are grateful to Lisa Mullins, Erin Belloli, Luann Linsalata, and Julie Kim for T cell subset assays. This work was supported by NIA grants AG11687 and AG11067 and by the University of Michigan's Claude Pepper Older Americans Independence Center (AG08808).

\section{REFERENCES}

1. Roberts-Thomson, I. C., Whittingham, S., Youngchaiyud, U., and Mackay, I. R. (1974) Ageing, immune response, and mortality. Lancet 2, 368-370

2. Murasko, D. M., Weiner, P., and Kaye, D. (1988) Association of lack of mitogen-induced lymphocyte proliferation with increased mortality in the elderly. Aging: Immunol. Infect. Dis. 1, 1-6

3. Wayne, S. J., Rhyne, R. L., Garry, P. J., and Goodwin, J. S. (1990) Cell-mediated immunity as a predictor of morbidity and mortality in subjects over 60 . J. Gerontol. Med. Sci. 45, M45-M48

4. Ferguson, F. G., Wikby, A., Maxson, P., Olsson, J., and Johansson, B. (1995) Immune parameters in a longitudinal study of a very old population of Swedish people: a comparison between survivors and nonsurvivors. J. Gerontol. A. Biol. Sci. Med. Sci. 50, B378B382

5. Ernst, D. N., Hobbs, M. V., Torbett, B. E., Glasebrook, A. L., Rehse, M. A., Bottomly, K., Hayakawa, K., Hardy, R. R., and Weigle, W. O. (1990) Differences in the expression profiles of CD45RB, Pgp-1, and 3G11 membrane antigens and in the patterns of lymphokine secretion by splenic $\mathrm{CD4}^{+} \mathrm{T}$ cells from young and aged mice. J. Immunol. 145, 1295-1302

6. Lerner, A., Yamada, T., and Miller, R. A. (1989) PGP-1 ${ }^{\text {hi }}$ T lymphocytes accumulate with age in mice and respond poorly to Concanavalin A. Eur. J. Immunol. 19, 977-982

7. Serra, H. M., Krowka, J. F., Ledbetter, J. A., and Pilarski, L. M. (1988) Loss of CD45R (Lp220) represents a post-thymic T cell differentiation event. J. Immunol. 140, 1435-1441

8. Pilarski, L. M., Yacyshyn, B. R., Jensen, G. S., Pruski, E., and Pabst, H. F. (1991) $\beta 1$ integrin (CD29) expression on human postnatal $T$ cell subsets defined by selective CD45 isoform expression. $J$. Immunol. 147, 830-837

9. Witkowski, J. M., and Miller, R. A. (1993) Increased function of P-glycoprotein in T lymphocytes of aging mice. J. Immunol. 150, 1296-1306

10. Witkowski, J. M., Li, S. P., Gorgas, G., and Miller, R. A. (1994) Extrusion of the P-glycoprotein substrate rhodamine-123 distinguishes CD4 memory $T$ cell subsets that differ in IL-2-driven II-4 production. J. Immunol. 153, 658-665

11. Bining, N., and Miller, R. A. (1997) Cytokine production by subsets of CD4 memory $\mathrm{T}$ cells differing in P-glycoprotein expression: effects of aging. J. Gerontol. A. Biol. Sci. Med. Sci. 52A, B137B145

12. Flurkey, K., Stadecker, M., and Miller, R. A. (1992) Memory T lymphocyte hyporesponsiveness to non-cognate stimuli: a key factor in age-related immunodeficiency. Eur.J. Immunol. 22, 931935

13. Chrisp, C. E., Turke, P., Luciano, A., Swalwell, S., Peterson, J., and Miller, R. A. (1996) Lifespan and pathology in genetically heterogeneous (four-way cross) mice: a new model for aging research. Vet. Pathol. 33, 735-743

14. Everitt, J. I., Ross, P. W., and Davis, T. W. (1988) Urologic syndrome associated with wire caging in AKR mice. Lab. Animal Sci. 38, 609-611

15. Tuffery, A. A. (1966) Urogenital lesions in laboratory mice. J. Pathol. Bacteriol. 91, 301-309

16. Miller, R. A., Bookstein, F., van der Meulen, J. H., Engle, S., Kim, J., Mullins, L., and Faulkner, J. (1996) Candidate biomarkers of aging: age-sensitive indices of immune and muscle function covary in genetically heterogeneous mice. J. Gerontol. Biol. Sci. 52A, B39-B47

17. Rao, C. R. (1973) Linear Statistical Inference and Its Applications, Wiley and Sons, New York

18. Boersma, W. J. A., Steinmeier, F. A., and Haaijman, J. J. (1985) Age-related changes in the relative numbers of Thy-1 and Lyt-2bearing peripheral blood lymphocytes in mice: a longitudinal approach. Cell. Immunol. 93, 417-430

19. Grossmann, A., Maggio-Price, L., Jinneman, J. C., and Rabinovitch, P. S. (1991) Influence of aging on intracellular free calcium and proliferation of mouse T-cell subsets from various lymphoid organs. Cell Immunol. 135, 118-131

20. Miller, R. A. (1997) Age-related changes in T cell surface markers: a longitudinal analysis in genetically heterogeneous mice. Mech. Ageing Dev. In press

21. Miller, R. A. (1995) Immune system. In Handbook of Physiology. Section 11: Physiology of Aging (Masoro, E., ed) pp. 555-590, Oxford University Press, New York 
22. Covelli, V., Mouton, D., Di Majo, V., Bouthillier, Y., Bangrazi, C., Mevel, J. C., Rebessi, S., Doria, G., and Biozzi, G. (1989) Inheritance of immune responsiveness, life span, and disease incidence in interline crosses of mice selected for high or low multispecific antibody production. J. Immunol. 142, 1224-1234

23. Miller, R. A., Turke, P., Chrisp, C., Ruger, J., Luciano, A., Peterson, J., Chalmers, K., Gorgas, G., and VanCise, S. (1994) Agesensitive $T$ cell phenotypes covary in genetically heterogeneous mice and predict early death from lymphoma. J. Gerontol. 49, B255-B262

24. Utsuyama, M., Hirokawa, K, Kurashima, C., Fukayama, M., Inamatsu, T., Suzuki, K., Hashimoto, W., and Sato, K. (1992) Differential age-change in the numbers of $\mathrm{CD}^{+} \mathrm{CD} 45 \mathrm{RA}^{+}$and $\mathrm{CD4}^{+} \mathrm{CD} 29^{+} \mathrm{T}$ cell subsets in human peripheral blood. Mech. Ageing Dev. 63, 57-68

25. Bronson, R. T. (1990) Rate of occurrence of lesions in 20 inbred and hybrid genotypes of rats and mice sacrificed at 6 month intervals during the first years of life. In Genetic Effects on Aging II (Harrison, D. E., ed) pp. 279-357, Telford Press, Caldwell, N.J.

26. Ershler, W. B. (1993) The influence of an aging immune system on cancer incidence and progression. J. Gerontol. Biol. Sci. 48, B3B7

27. Hirokawa, K, and Utsuyama, M. (1989) Combined grafting of bone marrow and thymus, and sequential multiple thymus graft- ings in various strains of mice. The effect on immune functions and life span. Mech. Ageing Dev. 49, 49-60

28. Baker, G. T., and Sprott, R. L. (1988) Biomarkers of aging. Exp. Gerontol. 23, 223-239

29. Masoro, E. J. (1995) Aging: current concepts. Handbook of Physiology. Section 11: Aging (Masoro, E. J., ed) Oxford University Press, New York

30. Costa, P. T., and McCrae, R. R. (1988) Measures and markers of biological aging: "a great clamoring. . of fleeting signifcance." Arch. Gerontol. Geriatr. 7, 211-214

31. Costa, P. T., and McCrae, R. R. (1995) Design and analysis of aging studies. In Handbook of Physiology. Section 11: Aging (Masoro, E. J., ed) Oxford University Press, New York

32. Nakamura, E., Lane, M. A., Roth, G. S., Cutler, R. G., and Ingram, D. K. (1994) Evaluating measures of hematology and blood chemistry in male rhesus monkeys as biomarkers of aging. Exp. Gerontol. 29, 151-177

33. Short, R., Williams, D. D., and Bowden, D. M. (1997) Circulating antioxidants as determinants of the rate of biological aging in pigtailed macaques (Macaca nemestrina). J. Gerontol. Biol. Sci. 52A, B26-B38

Received for publication March 12, 1997. Accepted for publication May 30, 1997. 